\title{
Tendências de busca na internet sobre cárie dentária e defeitos de desenvolvimento de esmalte no período de 2004 a 2015
}

\author{
Internet search trends about dental caries and developmental enamel defects, 2004-2015
}

\begin{abstract}
Natália Silva Andrade, ${ }^{1}$ Hélvis Enri de Sousa Paz, ${ }^{2}$ Teresinha Soares Pereira Lopes, ${ }^{2}$ Marcoeli Silva Moura, ${ }^{2}$ Lúcia de Fátima Almeida de Deus Moura, ${ }^{2}$ Marina de Deus Moura de Lima ${ }^{2}$

'Departamento de Estomatologia, Faculdade de Odontologia, Universidade de São Paulo, São Paulo, SP, Brasil

'Departamento de Patologia e Clínica Odontológica, Curso de Odontologia, Universidade Federal do Piauí, Teresina, PI, Brasil

- Os autores declaram que não há conflito de interesse.

\section{Resumo}

Objetivo: avaliar tendências de busca dos usuários da internet por informações sobre cárie dentária e defeitos de esmalte. Material e Métodos: trata-se de estudo retrospectivo que utilizou a ferramenta Google Trends Search Application como instrumento de busca. Foram utilizados os descritores "dental caries" e "enamel defects" para obtenção de informações no período de janeiro de 2004 e dezembro de 2015. Os resultados foram expressos pelo índice Search Volume Index. Foram utilizados os testes Kruskal-Wallis e pós-teste de Dunn, com nível de significância de $5 \%$, para a análise dos dados. Resultados: para o termo "dental caries", os maiores volumes de busca foram observados nos meses de março, abril e maio $(62.33 \pm 14.73,63.58 \pm 13.19,63.00 \pm 13.24$, respectivamente) e os menores, nos meses de julho, agosto e dezembro $(44.92 \pm 8.82,46.83 \pm 9.94,40.67 \pm 5.55)(p<0,001)$. Não foram observadas diferenças no volume de buscas entre meses do ano para o termo "enamel defects" $(p=0,694)$. $\mathrm{Na}$ análise por ano, foram observadas diferenças significativas para cárie dentária $(p<0,001)$, a partir de 2007 o volume de busca reduziu significativamente quando comparado aos anos anteriores. 0 mesmo não foi observado para defeitos de esmalte $(p=0,492)$. Conclusão: houve tendência de diminuição no número de buscas por informações sobre cárie dentária, ao passo que se mantêm constantes as buscas sobre defeitos de esmalte.
\end{abstract}

Palavras-chave: Cárie dentária; Esmalte dentário; Saúde bucal; Internet; Anormalidades dentárias.

\section{ABstRAct}

Objective: to assess the search trends among Internet users for information on dental caries and enamel defects. Material and Methods: this was a retrospective study that used the Google Search Trends Application as a search tool. The keywords "dental caries" and "enamel defects" were used to obtain information from January 2004 to December 2015. The results were expressed using the "search volume index." The Kruskal-Wallis test and Dunn's post-hoc test were used for data analysis, with a significance level of 5\%. Results: dental caries showed the highest search volumes in the months of March, April, and May (62.33 $\pm 14.73,63.58 \pm 13.19,63.00 \pm 13: 24$, respectively) and the lowest search volumes in July, August, and December (44.92 $\pm 8.82,46.83 \pm 9.94,40.67 \pm 5.55$, respectively) $(p<0.001)$, whereas no differences were observed in the search volumes for enamel defects $(p=0.694)$ during any of the months. Moreover, significant differences were observed for dental caries $(p<0.001)$ in 2007 , and the search volume reduced significantly when compared to previous years; however, different results were observed for enamel defects $(p=0.492)$. Conclusion: a descending trend was observed in the search volume for information on dental caries, whereas the search volume for enamel defects remained constant.

Keywords: Dental caries; Tooth enamel; Oral health; Internet; Tooth abnormalities.

\section{Introdução}

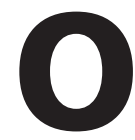
uso da Internet para a busca de informações relativas à saúde, principalmente sobre doenças específicas, sinais e sintomas, diagnóstico, tratamentos e possíveis efeitos adversos aumentou significativamente nos últimos anos. ${ }^{1,2}$

O número crescente de pesquisas online utilizando motores de busca populares fornece informações importantes sobre os diversos tipos de usuários e seus comportamentos diante das informações apreendidas. A análise desses dados resultou em nova área de investigação, denominada "infodemiologia", definida como ciência de determinantes e da distribuição da informação em meio eletrônico, especificamente a Internet, ou em uma população, com o objetivo de gerar dados úteis para saúde e políticas públicas. Os indicadores dessa área são gerados a partir de métodos para analisar pesquisa, comunicação e comportamento de publicação na Internet. ${ }^{3}$

O Google é a ferramenta de pesquisa mais utilizada por pacientes e profissionais para buscar informações online relacionadas à saúde. ${ }^{2}$ Dados de tendência gerados pelo número de buscas no Google ao longo do tempo podem ser analisados pelo serviço de acesso livre Google Trends. Essa ferramenta online permite aos usuários elaborarem gráficos de frequência de pesquisas para termos simples ou múltiplos. ${ }^{4}$ Os gráficos são obtidos normalizados em termos relativos e a análise pode ser feita em intervalos de tempo específicos ou regiões geográficas. ${ }^{4,5}$ Essa ferramenta tem sido utilizada principalmente para monitoramento de doenças infecciosas em tempo real. ${ }^{5}$ Além disso, o Google Trends apresenta potencial para ser utilizado como ferramenta para vigilância e pesquisas sobre diversos temas de saúde. ${ }^{6}$ No campo da Odontologia, apenas um estudo utilizou essa ferramenta como fonte de informação para analisar o comportamento online do volume de buscas na Internet sobre problemas bucais. ${ }^{7} \mathrm{O}$ uso de análise de dados na Internet neste campo é importante e oportuno, pois doenças bucais têm impacto negativo no bem-estar físico, social e psicológico da população em geral..$^{8-10}$

A cárie dentária permanece como a doença bucal crônica não transmissível de maior prevalência em seres humanos e sua evolução é a principal causa de dor e consequente perda dentária. ${ }^{11}$ Nos últimos anos, com o declínio de sua incidência e mudança no perfil epidemiológico ${ }^{12}$, os estudos têm abordado outras 
condições relacionadas à saúde bucal, destacando os defeitos de desenvolvimento de esmalte (DDE) ${ }^{13,14}$ Esses defeitos caracterizam-se pela diminuição local da translucidez do esmalte ou perda de sua estrutura ${ }^{15}$ e são considerados fatores de risco para o desenvolvimento da cárie dentária. ${ }^{13}$

Os motores de busca estão entre as mais populares páginas da web e tem como um de seus objetivos classificar outras páginas por relevância e popularidade. Assim podem auxiliar pacientes e profissionais a localizar informações de forma rápida e eficiente. Os dados de tendências de pesquisa na Internet representam uma forma fácil e viável de pesquisar a demanda por informações de saúde bucal, sendo útil aos mais diversos atores envolvidos (pacientes, profissionais e pesquisadores). Portanto, o presente estudo objetivou avaliar as tendências de busca dos usuários da Internet de todo o mundo por informações relacionadas à cárie dentária e a defeitos de desenvolvimento de esmalte, de modo a correlacionar esses dados à situação na qual se encontram tais patologias.

\section{Material e Métodos}

Estudo retrospectivo foi realizado utilizando-se a ferramenta Google Trends Search Application (disponível em: http://www. google.com/trends, acessado em 31 de outubro de 2015). Após investigação inicial, foram selecionados os descritores de pesquisa "dental caries" e "enamel defects", considerando que esses eram os termos com maior volume de pesquisa para gerar gráficos na ferramenta, sem a utilização de filtros. Foram obtidas informações sobre o volume de busca dos usuários da internet entre 01 de janeiro de 2004 e 31 de outubro de 2015 (disponíveis os resultados parciais para o mês de outubro de 2015 e incluídas as estimativas para os meses de novembro e dezembro de 2015).

Os resultados obtidos foram analisados em gráficos de tendência temporal de variação do índice SVI (Search Volume Index), disponibilizado pelo Google Trends. Essa ferramenta ajusta os dados de busca entre os termos, evitando que localidades com maior volume de pesquisa tenham sempre classificações mais altas. Para isso, cada ponto de dados é dividido pelas pesquisas totais por área geográfica e tempo que o representa, obtendose a comparação relativa da popularidade do termo no Google. Os números resultantes são dimensionados em escala de valores entre 0 (menor interesse de pesquisa) e 100 (maior interesse de pesquisa). ${ }^{4}$

O gráfico gerado no Google Trends apresenta no eixo horizontal o tempo (a partir de 2004, ano em que a ferramenta tornou-se disponível), e no eixo vertical quantas vezes o termo foi pesquisado em relação ao número total de buscas de acordo com o índice SVI. Google Trends também exibe manchetes de notícias relacionadas ao período da pesquisa, regiões e cidades com maior volume de buscas e informações sobre buscas relacionadas com base no volume de pesquisa do termo original. Embora relacionado à busca inicial, os termos fornecidos pela própria ferramenta rendem volumes de pesquisa menores ou resultados muito semelhantes. ${ }^{4}$ Esses termos também foram analisados para gerar gráficos de tendências de buscas.

A partir dos dados gerados pelo Google Trends, foi criado banco de dados para análises das tendências de buscas ao longo dos meses e dos anos e elaborado gráfico de dispersão (Gráfico 1) para os dois termos pesquisados ("dental caries" e "enamel defects").

Gráfico 1. Gráfico de dispersão para tendências temporais de busca dos termos "dental caries" e "enamel defects"

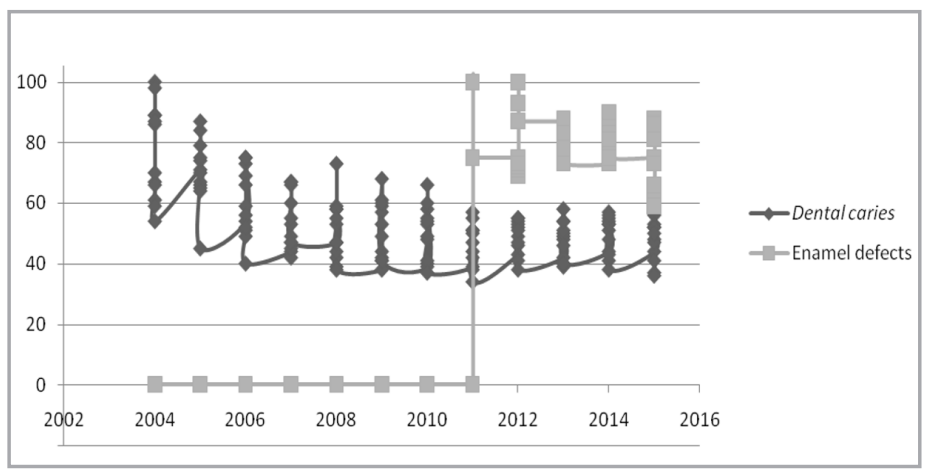

A análise estatística foi realizada no programa GraphPad Prism ${ }^{\oplus}$ versão 5.0 para Windows ${ }^{\circledR}$. Foi realizada análise descritiva dos dados para obtenção de medidas de tendência central (média) e de dispersão (desvio-padrão, valor mínimo e máximo). Foi aplicado o teste de Kolmogorov-Smirnov para testar a hipótese de normalidade, que teve distribuição não paramétrica. Assim, foram utilizados o teste de Kruskal-Wallis e pós-teste de Dunn para comparar a ordenação dos valores entre as variáveis analisadas, com nível de significância de 5\%.

\section{Resultados}

Foram analisadas as tendências de busca ao longo dos meses do ano sobre os termos "dental caries" e "enamel defects". No que se refere ao termo "dental caries", os maiores volumes de busca foram observados nos meses de março, abril, maio e outubro $(62.33 \pm 14.73,63.58 \pm 13.19,63.00 \pm 13.24,60.17 \pm 10.81$, respectivamente) e os menores nos meses de janeiro, julho, agosto e dezembro $(47.67 \pm 10.86,44.92 \pm 8.82,46.83 \pm 9.94,40.67 \pm 5.55$, respectivamente) $(\mathrm{p}<0,001)$ de todos os anos analisados (Tabela 1). Os índices médios dos meses de julho foram significativamente menores que os índices médios dos meses de fevereiro, março, abril, maio, setembro, outubro e novembro. Para o mês de dezembro, foram observadas associações para os meses de fevereiro, março, abril, maio, junho, setembro, outubro e novembro (Tabela 2). Não houve diferenças significativas no volume de buscas durante os meses do ano para o termo "enamel defects" (Tabela 1). 
Tabela 1. Tendências de buscas nos meses do ano sobre os termos "dental caries" e "enamel defects"

\begin{tabular}{l|c|c|c|c|c|c|c|c|c|c}
\hline \multicolumn{9}{|c}{ Dental caries } & \multicolumn{5}{c}{ Enamel defects } \\
\hline Mês & Méd. & DP & Mín. & Máx. & $\mathbf{p}^{*}$ & Méd. & DP & Mín. & Máx. & $\mathrm{p}^{*}$ \\
\hline Janeiro & 47,67 & 10,86 & 38 & 71 & $<0,001$ & 77,50 & 6,40 & 73 & 87 & 0,694 \\
\hline Fevereiro & 56,67 & 12,63 & 44 & 86 & & 78,50 & 5,74 & 75 & 87 & \\
\hline Março & 62,33 & 14,73 & 49 & 100 & & 76,50 & 6,61 & 69 & 83 & \\
\hline Abril & 63,58 & 13,19 & 51 & 89 & & 80,00 & 7,57 & 69 & 85 & \\
\hline Maio & 63,00 & 13,24 & 50 & 89 & & 82,00 & 8,98 & 69 & 88 & \\
\hline Junho & 51,00 & 7,54 & 42 & 67 & & 79,75 & 6,50 & 71 & 86 & \\
\hline Julho & 44,92 & 8,82 & 37 & 65 & & 80,50 & 6,03 & 72 & 86 & \\
\hline Agosto & 46,83 & 9,94 & 39 & 70 & & 81,25 & 5,68 & 73 & 86 & \\
\hline Setembro & 58,75 & 15,41 & 47 & 98 & & 88,00 & 8,63 & 78 & 100 & \\
\hline Outubro & 60,17 & 10,81 & 51 & 87 & & 85,75 & 11,50 & 75 & 100 & \\
\hline Novembro & 57,33 & 7,17 & 49 & 70 & & 80,25 & 5,12 & 75 & 87 & \\
\hline Dezembro & 40,67 & 5,55 & 34 & 54 & & 77,50 & 6,40 & 73 & 87 & \\
\hline TOTAL & 54,41 & 13,17 & 34 & 100 & & 80,78 & 7,29 & 69 & 100 & \\
\hline
\end{tabular}

Méd. - média; DP - desvio-padrão; Mín. - valor mínimo; Máx. - valor máximo. *Teste Kruskal Wallis

Tabela 2. Diferenças nas buscas mensais pelo termo "dental caries" pelo pós-teste de Dunn

\begin{tabular}{c|c|c|c|c|c|c|c|c|c|c|c|c}
\hline & JAN & FEV & MAR & ABR & MAI & JUN & JUL & AGO & SET & OUT & NOV & DEZ \\
\hline JAN & - & 0,052 & 0,002 & 0,001 & 0,001 & 0,469 & 0,550 & 0,856 & 0,017 & 0,007 & 0,037 & 0,129 \\
\hline FEV & 0,052 & - & 0,219 & 0,134 & 0,170 & 0,219 & 0,012 & 0,034 & 0,650 & 0,447 & 0,885 & 0,001 \\
\hline MAR & 0,002 & 0,219 & - & 0,786 & 0,885 & 0,015 & $<0,001$ & 0,001 & 0,436 & 0,637 & 0,278 & $<0,001$ \\
\hline ABR & 0,001 & 0,134 & 0,786 & - & 0,899 & 0,007 & $<0,001$ & $<0,001$ & 0,294 & 0,458 & 0,175 & $<0,001$ \\
\hline MAI & 0,001 & 0,170 & 0,885 & 0,899 & - & 0,100 & $<0,001$ & 0,001 & 0,356 & 0,538 & 0,219 & $<0,001$ \\
\hline JUN & 0,469 & 0,219 & 0,015 & 0,007 & 0,010 & - & 0,187 & 0,365 & 0,093 & 0,048 & 0,170 & 0,026 \\
\hline JUL & 0,550 & 0,012 & $<0,001$ & $<0,001$ & $<0,001$ & 0,187 & - & 0,677 & 0,003 & 0,001 & 0,008 & 0,356 \\
\hline AGO & 0,856 & 0,034 & 0,001 & $<0,001$ & 0,001 & 0,356 & 0,677 & - & 0,010 & 0,004 & 0,024 & 0,181 \\
\hline SET & 0,017 & 0,650 & 0,436 & 0,294 & 0,356 & 0,093 & 0,003 & 0,010 & - & 0,758 & 0,758 & $<0,001$ \\
\hline OUT & 0,007 & 0,447 & 0,637 & 0,458 & 0,538 & 0,048 & 0,001 & 0,004 & 0,758 & - & 0,538 & $<0,001$ \\
\hline NOV & 0,037 & 0,885 & 0,278 & 0,175 & 0,219 & 0,170 & 0,008 & 0,024 & 0,758 & 0,538 & - & $<0,001$ \\
\hline DEZ & 0,129 & 0,001 & $<0,001$ & $<0,001$ & $<0,001$ & 0,026 & 0,356 & 0,181 & $<0,001$ & $<0,001$ & 0,001 & - \\
\hline
\end{tabular}

$\mathrm{Na}$ análise das tendências de busca ao longo dos anos, foram observadas diferenças significativas $(\mathrm{p}<0,001)$ para o termo "dental caries" (Tabela 3). O volume de buscas sobre cárie dentária diminuiu significativamente ao longo dos anos (Tabela 4). Nos anos de 2004 e 2005, não houve diferenças significati- vas, entretanto, a partir de 2007 ( $\mathrm{p}<0,001)$ o volume de busca tem sido menor quando comparado aos anos anteriores. Já nas tendências de buscas anuais referentes ao termo "enamel defects" foi observado que os volumes de buscas permaneceram similares desde 2011 (Tabela 3). 
Tabela 3. Tendências de busca ao longo dos anos sobre os termos "dental caries" e "enamel defects"

\begin{tabular}{c|c|c|c|c|c|c|c|c|c|c}
\cline { 2 - 10 } & \multicolumn{5}{c|}{ Dental caries } & \multicolumn{4}{c}{ Enamel defects } \\
\hline $\mathbf{A n o}$ & Méd. & DP & Mín. & Máx. & $\mathbf{p}^{* *}$ & Méd. & DP & Mín. & Máx. & $\mathbf{p}^{* *}$ \\
\hline $\mathbf{2 0 0 4}$ & 77,17 & 16,01 & 54 & 100 & $<0,001$ & & & & & \\
\hline $\mathbf{2 0 0 5}$ & 70,58 & 10,93 & 45 & 87 & & - & - & - & - & - \\
\hline $\mathbf{2 0 0 6}$ & 59,92 & 11,50 & 40 & 75 & & - & - & - & - & - \\
\hline $\mathbf{2 0 0 7}$ & 53,50 & 9,36 & 42 & 67 & & - & - & - & - & - \\
\hline $\mathbf{2 0 0 8}$ & 51,08 & 10,18 & 38 & 73 & & - & - & - & - & - \\
\hline $\mathbf{2 0 0 9}$ & 51,08 & 9,89 & 38 & 68 & & - & - & - & - & - \\
\hline $\mathbf{2 0 1 0}$ & 50,00 & 9,27 & 37 & 66 & & - & - & - & - & - \\
\hline $\mathbf{2 0 1 1}$ & 46,58 & 7,18 & 34 & 57 & & 81,25 & 12,50 & 75 & 100 & 0,492 \\
\hline $\mathbf{2 0 1 2}$ & 48,67 & 5,71 & 38 & 55 & & 78,33 & 10,62 & 69 & 100 & \\
\hline $\mathbf{2 0 1 3}$ & 47,58 & 6,27 & 39 & 58 & & 81,67 & 4,62 & 73 & 88 & \\
\hline $\mathbf{2 0 1 4}$ & 49,42 & 6,43 & 38 & 57 & & 81,42 & 4,58 & 73 & 90 & \\
\hline $\mathbf{2 0 1 5 *}$ & 47,33 & 6,51 & 36 & 56 & & 81,78 & 5,91 & 73 & 88 & \\
\hline TOTAL & 54,41 & 13,17 & 34 & 100 & & 80,78 & 7,29 & 69 & 100 & \\
\hline
\end{tabular}

Méd. - média; DP - desvio-padrão; Mín. - valor mínimo; Máx. - valor máximo. * Resultados parciais para mês de outubro de 2015 e estimativa de novembro e dezembro de 2015. **Teste Kruskal Wallis

Tabela 4. Diferenças nas buscas anuais pelo termo "dental caries" pelo pós-teste de Dunn

\begin{tabular}{c|c|c|c|c|c|c|c|c|c|c|c|c} 
& $\mathbf{2 0 0 4}$ & $\mathbf{2 0 0 5}$ & $\mathbf{2 0 0 6}$ & $\mathbf{2 0 0 7}$ & $\mathbf{2 0 0 8}$ & $\mathbf{2 0 0 9}$ & $\mathbf{2 0 1 0}$ & $\mathbf{2 0 1 1}$ & $\mathbf{2 0 1 2}$ & $\mathbf{2 0 1 3}$ & $\mathbf{2 0 1 4}$ & $\mathbf{2 0 1 5}$ \\
\hline $\mathbf{2 0 0 4}$ & - & 0,386 & 0,014 & 0,001 & $<0,001$ & $<0,001$ & $<0,001$ & $<0,001$ & $<0,001$ & $<0,001$ & $<0,001$ & $<0,001$ \\
\hline $\mathbf{2 0 0 5}$ & 0,386 & - & 0,219 & 0,001 & $<0,001$ & $<0,001$ & $<0,001$ & $<0,001$ & $<0,001$ & $<0,001$ & $<0,001$ & $<0,001$ \\
\hline $\mathbf{2 0 0 6}$ & 0,001 & 0,219 & - & 0,887 & 0,503 & 0,503 & 0,320 & 0,037 & 0,157 & 0,078 & 0,239 & 0,065 \\
\hline $\mathbf{2 0 0 7}$ & $<0,001$ & 0,001 & 0,887 & - & 1,000 & 1,000 & 0,999 & 0,827 & 0,984 & 0,932 & 0,996 & 0,912 \\
\hline $\mathbf{2 0 0 8}$ & $<0,001$ & $<0,001$ & 0,503 & 1,000 & - & 1,000 & 1,000 & 0,991 & 1,000 & 0,999 & 1,000 & 0,998 \\
\hline $\mathbf{2 0 0 9}$ & $<0,001$ & $<0,001$ & 0,503 & 1,000 & 1,000 & - & 1,000 & 0,991 & 1,000 & 0,999 & 1,000 & 0,998 \\
\hline $\mathbf{2 0 1 0}$ & $<0,001$ & $<0,001$ & 0,320 & 0,999 & 1,000 & 1,000 & - & 0,999 & 1,000 & 1,000 & 1,000 & 1,000 \\
\hline $\mathbf{2 0 1 1}$ & $<0,001$ & $<0,001$ & 0,037 & 0,827 & 0,991 & 0,991 & 0,999 & - & 1,000 & 1,000 & 1,000 & 1,000 \\
\hline $\mathbf{2 0 1 2}$ & $<0,001$ & $<0,001$ & 0,157 & 0,984 & 1,000 & 1,000 & 1,000 & 1,000 & - & 1,000 & 1,000 & 1,000 \\
\hline $\mathbf{2 0 1 3}$ & $<0,001$ & $<0,001$ & 0,078 & 0,932 & 0,999 & 0,999 & 1,000 & 1,000 & 1,000 & - & 1,000 & 1,000 \\
\hline $\mathbf{2 0 1 4}$ & $<0,001$ & $<0,001$ & 0,239 & 0,996 & 1,000 & 1,000 & 1,000 & 1,000 & 1,000 & 1,000 & - & 1,000 \\
\hline $\mathbf{2 0 1 5 *}$ & $<0,001$ & $<0,001$ & 0,065 & 0,912 & 0,998 & 0,998 & 1,000 & 1,000 & 1,000 & 1,000 & 1,000 & - \\
\hline
\end{tabular}

O gráfico 1 demonstra a análise temporal em gráfico de dispersão para os dois termos de busca. Quanto à localização, notícias e pesquisas relacionadas aos termos "dental caries" e "enamel defects", verificou-se que para defeitos de esmalte os volumes de busca ainda não são suficientes para maiores esclarecimentos. Houve destaque para publicação do The Indu, em fevereiro de 2010, sobre estudo relacionando DDE e mortalidade precoce. Para cárie den- tária, os países com maiores volumes de buscas foram Peru (SVI $=100)$, seguido da Venezuela (SVI = 71) e Equador (SVI = 59). As notícias em destaque no Google para cárie dentária foram sobre conhecimento científico da etiologia da cárie (Doctor NDTV em novembro de 2012), predisposição genética da doença (One India - em setembro de 2010) e guia para prevenção de cárie (Nature.com - em outubro de 2008 - Quadro 1). 
Quadro 1. Regiões, cidades, notícias e termos mais populares que foram procurados por amplo tópico de pesquisa

\begin{tabular}{|c|c|c|c|c|}
\hline $\begin{array}{l}\text { Tópicos de } \\
\text { Pesquisa }\end{array}$ & $\begin{array}{l}\text { Ranque de } \\
\text { Regiões }\end{array}$ & $\begin{array}{l}\text { Ranque de } \\
\text { Cidades }\end{array}$ & $\begin{array}{c}\text { Pesquisas } \\
\text { Relacionadas }\end{array}$ & $\begin{array}{l}\text { Principais } \\
\text { notícias }\end{array}$ \\
\hline $\begin{array}{l}\text { Dental } \\
\text { caries }\end{array}$ & \begin{tabular}{|l|} 
Peru - 100 \\
Venezuela - 71 \\
Equador - 59
\end{tabular} & $\begin{array}{l}\text { La Paz - } 100 \\
\text { La Victoria - } 50 \\
\text { Breña - } 44\end{array}$ & \begin{tabular}{|l} 
Dental car- \\
ies-syndrome \\
-100 \\
Dental la caries \\
-100 \\
La caries - 100 \\
Dental caries \\
treatment - 30
\end{tabular} & $\begin{array}{l}\text { - Doctor NDTV } \\
\text { - em novembro } \\
\text { de } 2012 \text { - Dental } \\
\text { caries: The science } \\
\text { behind what } \\
\text { causes our tooth } \\
\text { decay? } \\
\text { - One India - em } \\
\text { setembro de } 2010 \\
\text { - Taste genes pre- } \\
\text { dict dental caries. } \\
\text { - Nature.com -em } \\
\text { outubro de } 2008 \\
\text { - Vital guide to } \\
\text { preventing dental } \\
\text { caries. }\end{array}$ \\
\hline $\begin{array}{l}\text { Enamel } \\
\text { defects }\end{array}$ & $\begin{array}{l}\text { Dados não } \\
\text { gerados }\end{array}$ & $\begin{array}{l}\text { Dados não } \\
\text { gerados }\end{array}$ & $\begin{array}{l}\text { Dental enamel } \\
\text { defects - } 100 \\
\text { Tooth enamel } \\
\text { defects - } 85\end{array}$ & $\begin{array}{l}\text { - The Indu - em } \\
\text { fevereiro de } 2010 \\
\text {-Enamel defects } \\
\text { linked to early } \\
\text { mortality: study. }\end{array}$ \\
\hline
\end{tabular}

\section{Discussão}

O uso da Internet pela população em geral possui inúmeras vantagens, como facilidade de acesso à informação e comunicação. Essa ferramenta tornou-se uma fonte nova, livre e gratuita de disseminação de informações na área da saúde..$^{16}$ Google Trends tem sido utilizado tanto na produção de conhecimento para inferência causal, descrição e vigilância de problemas de saúde quanto no estudo de fenômenos de saúde. ${ }^{5}$ Nesta pesquisa, essa ferramenta foi selecionada pela possibilidade de avaliar tendências de buscas dos usuários da internet sobre informações de cárie dentária e defeitos de desenvolvimento de esmalte. No entanto, o uso do Google Trends precisa estabelecer métodos reprodutíveis a fim de determinar a consistência dos resultados fornecidos para realização de consultas ao longo do tempo. ${ }^{5}$ Para minimizar essa limitação na utilização da ferramenta, os pesquisadores deste estudo selecionaram termos específicos e que geraram maior volume de buscas para análise dos resultados.

O Google Trends permite análise transversal de pesquisas na Web do Google. Essa análise indica a probabilidade de um usuário típico pesquisar por um termo específico a partir de um determinado local em um determinado momento. O sistema também elimina consultas repetidas de um único utilizador durante um curto período de tempo, para que os dados não sejam aumentados artificialmente. $^{7}$

Buscas sobre "dental caries" foram mais comumente encontradas em países em desenvolvimento latino-americanos. Cárie dentária é uma das doenças crônicas mais prevalentes em seres humanos e a dor ocasionada é a complicação mais frequente e principal fonte de queixas dos pacientes. ${ }^{17}$ Há evidência da diminuição da prevalência dessa doença em crianças da América Latina e Caribe, embora essa tenha sido menos proeminente nos últimos anos. ${ }^{18}$ Em países como o Peru, a prevalência de problemas de saúde bucal em crianças, como cárie dentária não tratada, permanece alta. ${ }^{19,20}$ Essas características da doença e sua epidemiologia justificariam o maior interesse dos usuários dessa região em buscar informações sobre cárie dentária.
A introdução de fluoretos em suas diversas formas, associada à melhora nos hábitos de higiene bucal e à redução do consumo de sacarose justificam o declínio dos índices de cárie. ${ }^{12,18}$ Esses fatores poderiam também justificar a diminuição do volume de buscas sobre o tema ao longo dos anos. Outras razões, como mudanças nos critérios de diagnóstico, maior acesso a serviços odontológicos e maior disponibilidade de informações à população sobre o tema. ${ }^{18,21}$ As buscas relacionadas à cárie dentária diminuíram nos meses de janeiro, julho, agosto e dezembro, correspondentes aos períodos de férias de muitos países, nos quais os internautas buscam informações mais relacionadas a outros assuntos de interesse no período.

Os defeitos de desenvolvimento do esmalte dentário provocam distúrbios na mineralização ou perda de estrutura dentária, que podem ocasionar dor, comprometimento estético e perda de função, ${ }^{22,23} \mathrm{o}$ que pode influenciar na percepção do indivíduo sobre sua saúde bucal e interesse em buscar informações sobre o problema que lhe aflige.

Não foi observada a mesma tendência das buscas sobre informações relativas à cárie dentária durante os meses do ano para os defeitos de esmalte. Esse resultado pode ser explicado, pois os volumes de busca para o termo só foram considerados significativos pelo índice SVI a partir do ano de 2011.

Cerca de um quarto de pacientes de consultórios odontológicos buscam informações sobre saúde bucal. ${ }^{24} \mathrm{O}$ aumento do uso da Internet para pesquisar informações sobre saúde ${ }^{25}$ pode ampliar o conhecimento do usuário, auxiliando-o a gerir melhor o processo saúde-doença e permitindo discutir melhores opções de tratamento, reduzindo a ansiedade frente aos problemas de saúde. ${ }^{16}$

Nesse contexto, a ampliação e democratização do acesso às informações em saúde na Internet tem causado impacto significativo na relação profissional de saúde/paciente, pois esse profissional passa a lidar com o conhecimento adquirido pelo paciente, até então restrito a especialistas na área. ${ }^{1}$ No entanto, diante da diversidade de informações disponíveis na Internet, os usuários necessitam de orientação para avaliar a confiabilidade das mesmas. Cirurgiões-dentistas devem estar preparados para discutir questões levantadas pelos pacientes sobre informações obtidas online. E, atualmente, atenção dever ser direcionada à compra de produtos de saúde bucal na Internet que se torna cada vez mais comum. ${ }^{25}$

A Health on Net Foundation (HON) elaborou um código de condutas (HONCode) para veicular informações na internet sobre saúde de maneira ética e proteger os cidadãos de informações de saúde enganosas, entretanto, no Brasil, são poucos os sites creditados pela HON. ${ }^{1} \mathrm{Na}$ busca por informações em saúde, uma série de preocupações também são geradas acerca da credibilidade, qualidade e regulação, podendo se constituir num risco para a saúde pública inclusive no âmbito odontológico. ${ }^{21}$

As limitações deste estudo incluem a necessidade de utilização de descritores em inglês. Além disso, a informação da Internet é dinâmica e sujeita a mudanças diárias, de acordo com a mudança do comportamento humano, de modo que os métodos utilizados não podem medir de forma confiável os níveis de doença, embora os profissionais de saúde bucal possam monitorar as tendências para ajudar no planejamento, marketing e comunicação sobre 
problemas bucais. O método de pesquisa de consulta também pode ser usado para detectar e investigar novas doenças, examinando queixas específicas e mudanças súbitas em uma região local, embora este método necessite de mais investigação.?

\section{Conclusão}

Foi observada tendência de queda no volume global de pesquisas sobre cárie dentária, ao passo que se mantêm constantes os volumes de busca sobre defeitos de desenvolvimento de esmalte.

\section{Referências}

1. Moretti FA, Oliveira VE, Silva EMK. Acesso a informações de saúde na internet: uma questão de saúde pública? Rev Assoc Med Bras. 2012;58(6):650-8.

2. Brigo F, Trinka E. Google search behavior for status epilepticus. Epilepsy Behav 2015; http://dx.doi.org/10.1016/j.yebeh.2015.02.029.

3. Eysenbach G. Infodemiology and infoveillance tracking online health information and cyberbehavior for public health. Am J Prev Med. 2011;40(5):S154-8.

4. Google Trends. https://support.google.com/trends/topic/13975?hl=en\&ref_ topic $=$

13762; 2015. [accessed 31th October 2015].

5. Nuti SV, Wayda B, Ranasinghe I, Wang S, Dreyer RP, Chen SI, et al. The Use of Google Trends in Health Care Research: A Systematic Review. PLoS ONE. 2014;9(10):e109583. doi:10.1371/journal.pone.0109583.

6. Dehkordy SF, Carlos RC, Hall KS, Dalton VK. Novel Data Sources for Women's Health Research. Acad Radiol. 2014;21(9):1172-6.

7. Harorli OT, Harorli H. Evaluation of internet search trends of some common oral problems, 2004 to 2014. Community Dental Health. 2014;31:188-92.

8. Kramer PF, Feldens CA, Ferreira SH, Bervian J, Rodrigues PH, Peres MA. Exploring the impact of oral diseases and disorders on quality of life of preschool children. Community Dent Oral Epidemiol. 2013;41:327-35.

9. Brasil. Ministério da Saúde. Secretaria de Atenção à Saúde. Secretaria de Vigilância em Saúde. SB Brasil 2010: Pesquisa Nacional de Saúde Bucal: resultados principais/Ministério da Saúde. Secretaria de Atenção à Saúde. Secretaria de Vigilância em Saúde. - Brasília : Ministério da Saúde, 2012. 116 p.

10. Sischo L, Broder HL. Oral health-related quality of life: what, why, how and future. J Dent Res. 2011;90:1264-70.

11. Frazão P. Epidemiology of dental caries: when structure and context matter. Braz Oral Res. 2012;26(1):108-14.

12. Bönecker M, Cleaton-Jones P. Trends in dental caries in latin America and Caribbean 5-6 and 11-13-year-old children: a systematic review. Community Dent Oral Epidemiol. 2003;31:152-7.

13. Vargas-Ferreira F, Salas MMS, Nascimento GG, Tarquinio SBC, Faggion Jr CM, Peres MA. Association between developmental defects of enamel and dental caries: A systematic review and meta-analysis. J Dent. 2015;43:619-28.
14. Jacobsen PE, Haubek D, Henriksen TB, Ostergaard JR, Poulsen S. Developmental enamel defects in children born preterm: a systematic review. Eur J Oral Sci. 2014;122:7-14.

15. Federation Dentaire Internationale. A review of the developmental defects of dental index (DDE index). Commission on Oral Health Research and Epidemiology. Report of an FDI Working Group. Int Dent J. 1992;42:411-26.

16. Samadbeik M, Ahmadi M, Mohammadi A, Saravi BM. Health Information on Internet: Quality, Importance, and Popularity of Persian Health Websites. Iran Red Crescent Med J. 2014;16(4):e12866.

17. Shqair AQ, Gomes GB, Oliveira A, Goettems ML, Romano AR, Schardozim LR, et al. Dental emergencies in a university pediatric dentistry clinic: a retrospective study. Brazilian Oral Research. 2012;26:50-6.

18. Sofola OO, Folayan MO, Oginni AB. Changes in the prevalence of dental caries in primary school children in Lagos State, Nigeria. Niger J Clin Pract. 2014;17:127-33.

19. Delgado-Angulo EK, Hobdell MH, Bernabé E. Poverty, social exclusion and dental caries of 12-year-old children: A cross-sectional study in Lima, Peru. BMC Oral Health. 2009;9(1):16.

20. Pulache J, Abanto J, Oliveira LB, Bönecker M, Porras JCE. Exploring the association between oral health problems and oral health-related quality of life in Peruvian 11- to 14-year-old children. Int J Paediatr Dent. 2016;26(2):81-90.

21. Leite F, Correia A. Quality evaluation of websites with information on childhood dental caries in Portuguese language. Rev Odonto Cienc. 2011;26(2):11620.

22. Salanitri S, Seow WK. Developmental enamel defects in the primary dentition: aetiology and clinical management. Australian Dent J. 2013;58:133-40.

23. Seow W. Developmental defects of enamel and dentine: challenges for basic science research and clinical management. Australian Dent J. 2014;59(1):143-54. 24. Naganandinia S, Raob R, Kulkarnic SB. Survey on the Use of the Internet as a Source of Oral Health Information Among Dental Patients in Bangalore City, India. Oral Health Prev Dent. 2014;2:141-7.

25. Bujnowska-Fedak MM. Trends in the use of the Internet for health purposes in Poland. BMC Public Health. 2015; 15:194. DOI 10.1186/s12889-015-1473-3.

\section{Mini Currículo e Contribuição dos Autores}

1. Natália Silva Andrade - cirurgiã-dentista e mestre. Contribuição: concepção e delineamento, aquisição dos dados, interpretação dos dados, análise estatística, preparação e redação do manuscrito.

2. Hélvis Enri de Sousa Paz - acadêmico de Odontologia. Contribuição: aquisição dos dados, interpretação dos dados e redação do manuscrito.

3. Teresinha Soares Pereira Lopes - cirurgiã-dentista e PhD. Contribuição: revisão crítica e aprovação final.

4. Marcoeli Silva Moura - cirurgiã-dentista e PhD. Contribuição: revisão crítica e aprovação final.

5. Lúcia de Fátima Almeida de Deus Moura - cirurgiã-dentista e PhD. Contribuição: revisão crítica e aprovação final.

6.Marina de Deus Moura de Lima - cirurgiã-dentista e PhD. Contribuição: concepção e delineamento, revisão crítica e aprovação final.

Recebido em: 10/02/2017 / Aprovado em: 07/03/2017

Autor Correspondente

Marina de Deus Moura de Lima

E-mail: mdmlima@gmail.com 